\title{
Segurança na Internet das Coisas: Varredura, Análise e Estatística de Redes sem Fio em Bom Jesus do Itabapoana
}

\author{
Ana Julia B. Alves', Wesley Folly V. de Souza ${ }^{2}$ \\ ${ }^{1}$ Instituto Federal Fluminense - Bom Jesus do Itabapoana - RJ - Brasil \\ ${ }^{2}$ Coordenação de Informática - Instituto Federal Fluminense - Bom Jesus do Itabapoana \\ $-\mathrm{RJ}$ - Brasil \\ \{anajuliabritesalves, wesleyfolly\}@gmail.com
}

\begin{abstract}
In this work we will do a research with the aid of the wardriving method so that we can find out how much Wi-Fi networks in our region are vulnerable to attacks. To this end, we will capture and analyze data such as: security protocols, wps configurations, radio channels, device discrimination, among others.

Resumo. Neste trabalho foi realizada uma pesquisa com o auxílio do método wardriving para que possamos descobrir o quanto as redes $W i$ - $f i$ da região estão vulneráveis a ataques. Com esse objetivo, foi realizada a captura e análise de dados como: protocolos de segurança, configurações wps, canais de rádio, discriminação de dispositivos, entre outros.
\end{abstract}

\section{Introdução}

As redes sem fio apresentam um problema em relação às redes cabeadas: as vulnerabilidades e riscos são maiores, pois suas ondas eletromagnéticas atravessam o ar, paredes e muitos obstáculos tornando-se (caso a segurança da rede não seja muito bem planejada) um alvo perfeito para captura de informações de acordo com Oliveira (2010).

Diversos ataques podem ser feitos contra redes e dispositivos sem fio, especialmente se o atacante tiver acesso a informações como status da configuração WPS (Wi-Fi Protected Setup) do ponto de acesso, redes preferenciais de dispositivos IoT (Internet of Things) e protocolos de segurança e criptografia da rede, como WPA(Wireless Protected Access), WEP(Wired Equivalent Privacy) e WPA2 (Wireless Protected Access II).

Com o advento e popularização de dispositivos IoT, a rede Wireless se torna um ponto crítico na segurança destes dispositivos pois a maioria utiliza apenas essa forma de transmissão de dados. Por se conectarem principalmente a redes locais, a segurança implementada nesses dispositivos é fraca. Portanto, o comprometimento das redes sem fio deixa todos os dispositivos conectados a elas vulneráveis segundo Zabadal e Castro (2017). 


\section{Método}

Os equipamentos utilizados foram: um automóvel (figura 1), uma antena externa acoplada a ele (figura 1), um telefone celular da marca LG com Sistema Operacional Android, um notebook Lenovo com dual boot dos Sistemas Operacionais Windows $10 \mathrm{e}$ o Ubuntu 17.10. Foi utilizado o software Aircrack- $N G$ [aircrack-ng.org] no sistema Ubuntu (figura 2) e o aplicativo WiGLE-Wifi [wigle.net] no aparelho celular.

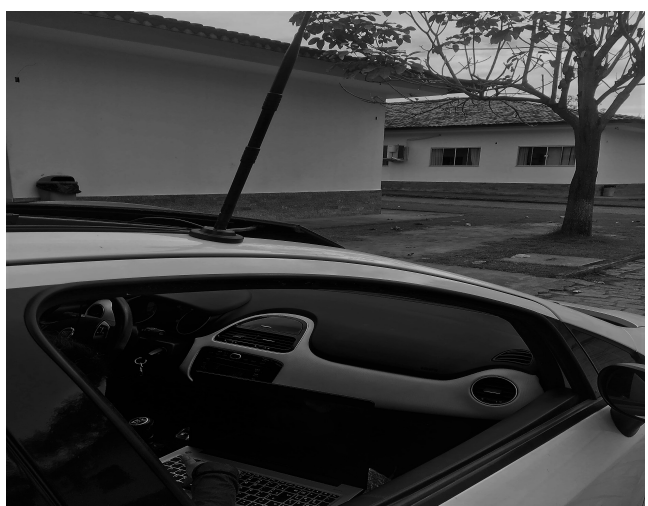

Figura 1. Antena e veículo utilizado na varredura.

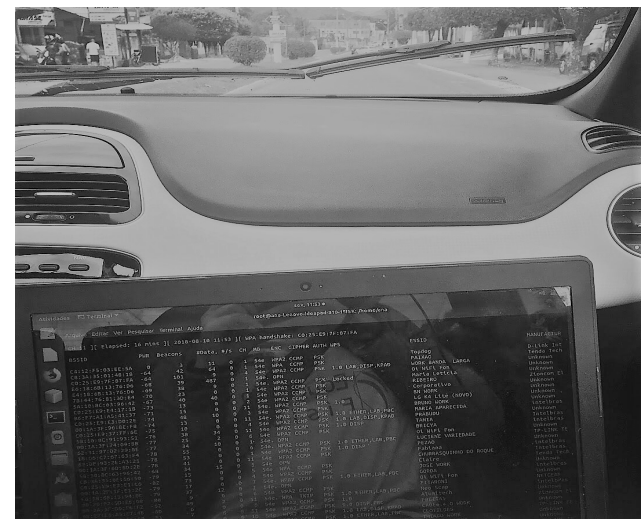

Figura 2. Software utilizado na varredura.

A varredura ocorreu no dia 10/08/2018, a mesma se iniciou no Instituto Federal Fluminense de Bom Jesus do Itabapoana e terminou nesse mesmo ponto, visto que foi feito um trajeto de retorno, totalizando um deslocamento de aproximadamente $5 \mathrm{Km}$.

A figura 3 mostra a distribuição desses pontos de acesso sobrepostos ao mapa da cidade de Bom Jesus do Itabapoana disponibilizado pelo Google Earth [Google].

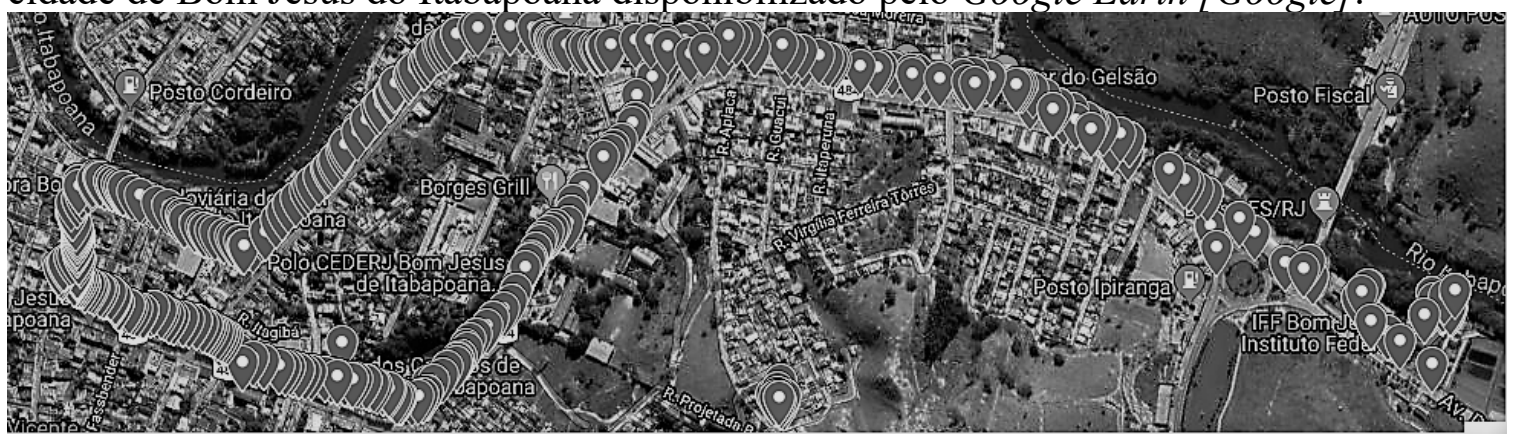

Figura 3. Distribuição de pontos de acesso na zona comercial da cidade de Bom Jesus do Itabapoana.

\section{Análise dos resultados}

O Aicrack-NG conseguiu capturar dados de aproximadamente 850 redes Wireless durante a varredura. Os dados escolhidos para estatística e análise foram: protocolos de 
segurança, status do WPS e visibilidade de redes preferenciais nos dispositivos.

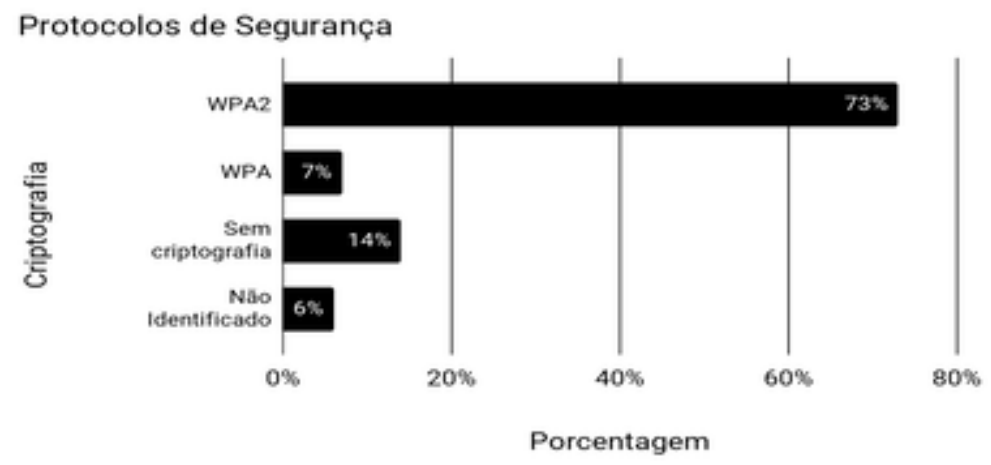

Figura 4. Protocolos de Segurança.

A figura 4 mostra que $73 \%$ das redes sem fio locais estão protegidas, pois usam o protocolo WPA2, porém 7\% possui o protocolo WPA, que não é tão seguro como dito por Lashkari, Danesh e Samadi (2009) e 14\% das redes não usam criptografia alguma, ou seja, os dados dos usuários da rede ficam totalmente expostos a invasores.

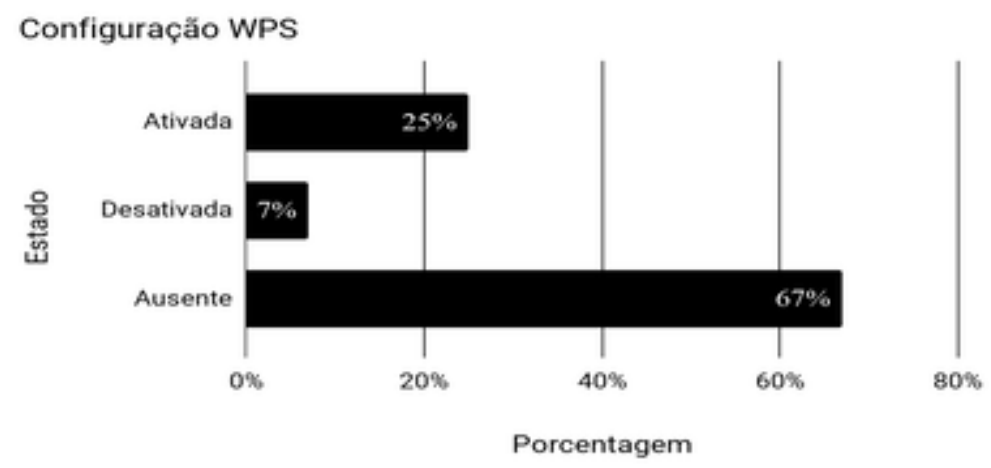

Figura 5. Ocorrência de ativação da configuração WPS.

Segundo a figura 5, a WPS não foi encontrada em muitas redes, o que significa que roteadores atuais estão deixando de ter essa opção habilitada por padrão, ou até mesmo que não usem mais ela. Mas, $25 \%$ estão com a WPS ativada, deixando a rede vulnerável mesmo que esta utilize o WPA2 e uma senha forte segundo Silva (2014).

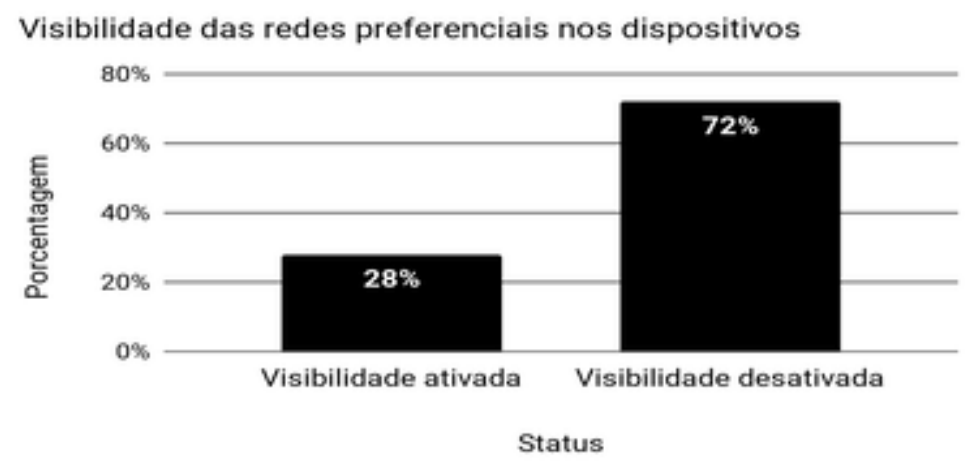


Figura 6. Status da visibilidade das redes preferenciais nos dispositivos.

Conforme a figura 6 , é possível notar que a maioria dos dispositivos ocultam suas redes preferenciais, o que é um ótimo indicativo de segurança. Mas, o número de usuários conectados às redes sem fio que foram rastreados pela ferramenta foi menor devido ao deslocamento do automóvel, portanto não se pode afirmar que essa índice é confiável.

Vale ressaltar que apenas redes fixas foram analisadas, não incluindo, portanto, redes $3 \mathrm{G}$ e $4 \mathrm{G}$.

\section{Conclusão}

Dado o exposto pode-se perceber que os dados relacionados à segurança dos dispositivos e redes da cidade são promissores, acredita-se que isso se deve ao fato de ser uma zona predominantemente comercial e portanto os usuários terem uma preocupação maior com a segurança e dessa forma terem posse de equipamentos modernos e configurados cuidadosamente por mão de obra especializada, porém ainda há vulnerabilidades que devem ser corrigidas para evitar furto de dados não autorizados.

\section{Referências}

OLIVEIRA, A. T. (2010) "Análise das Vulnerabilidades das Redes Sem Fio na Cidade de Vitória da Conquista-BA",73 f. TCC (Graduação) - Curso de Ciência da Computação, Ciências Exatas, Universidade Estadual do Sudoeste da Bahia, Vitória da Conquista - Ba , http://www2.uesb.br/computacao/wp-content/uploads/2014/09/ ANÁLISE-DAS-VULNERABILIDADES-DAS-REDES-SEM-FIO-NA-CIDADEDE-VITÓRIA-DA-CONQUISTA-BA-Alan-Teixeira-de-oliveira.pdf.

Arash Habibi Lashkari, Mir Mohammad Seyed Danesh and B. Samadi (2009), "A survey on wireless security protocols (WEP, WPA and WPA2/802.11i)," 2nd IEEE International Conference on Computer Science and Information Technology, Beijing, pp. 48-52.

SILVA, Cristiano de Souza (2014). VULNERABILIDADE DO WPS (WI-FI PROTECTED SETUP) NAS REDES SEM FIO. 20 f. Artigo - Curso de Perícia Digital, Universidade Católica de Brasília, Brasília. Disponível em: https://sistemas.stf.jus.br/dspace/xmlui/bitstream/handle/123456789/1189/

Monografia $\% 20-\% 20$ Cristiano\%20de\%20Souza\%20e\%20Silva.pdf? sequence $=1 \&$ isAllowed $=y$. Acesso em: 01 mar. 2020.

ZABADAL, Bernardo Moreira; CASTRO, Bianca Francinny Lisboa Murta de (2017). IoT e Seus Principais Desafios. 10 f. Artigo. Instituto Federal de Educação, Ciência e Tecnologia de São Paulo, São Paulo.Disponível em: http://rinte.ifsp.edu.br/index.php/RInTE/article/view/333/pdf_94. Acesso em: 01 mar. 2020. 